\title{
Spatial Embedding as an Enabling Constraint: Introduction to a Special Issue of Complexity on the topic of "Spatial Organisation"
}

\author{
Seth Bullock, Nicholas Geard
}

School of Electronics and Computer Science, University of Southampton, UK

\begin{abstract}
We introduce and discuss the role of spatial embedding as an enabling constraint on complex system structure and function.
\end{abstract}

Key words:

complexity, space, spatial embedding, networks,

\section{Space as an Enabling Constraint}

The vast majority of real-world complex systems are extended in space. Some parts are close together, others are far apart. Moreover, the tendency for particular system parts to interact is partly determined by the distances that separate them. Consequently, there is considerable evidence that this spatial embedding has a significant impact on both the structural and functional organisation of complex systems. Spatial constraints are implicated in the way that populations of termites construct their impressive mounds $[15,16]$, the way in which species of plants compete for light [8], and even the way in which genes are packed onto linear strands of DNA [18]. Moreover, we are beginning to understand that this influence is not neutral, but can sometimes be positive, with spatially embedded systems enjoying an improved ability to effectively organise in order to support useful functionality.

Key examples are offered by [5] in their work modelling the tendency of molecules to organise into mutually self-reinforcing "hypercycles" [11], and by [9] in the context of trying to understand how biological populations of simple creatures might evolve to exhibit co-operative tendencies (see [6], this volume, for more details of both models). In each case, the system being 
modelled was unable to exhibit organised complex behaviour when its constituent parts were well-mixed. However, when each system was extended over a two-dimensional space such that its parts could only interact with their close spatial neighbours, the systems were able to spontaneously achieve and maintain functional organisation in the face of exploitative disturbance. Spatial embedding enabled each population (of modelled molecular species or simple simulated creatures) to structure itself in such a way as to maintain co-operative functional organisation through exploiting the "useful" asymmetries that space introduces into the ecology of interactions [7].

These results suggest an intriguing possibility: might understanding the role of spatial embedding help us to better deal with problems concerning a wide range of spatially embedded complex systems? For instance, how to organise the built environment such that community coherence is consolidated rather than fragmented [4]; how to arrange social care processes such that they are vital and integrated [17]; how to engineer accountability in virtual communities [12]; how to achieve bioremediation using bacterial biofilm communities [19]; and how to improve the resilience of the infrastructural systems upon which we rely [10], etc. Could the influence of spatial embedding in these contexts, when properly understood, allow and encourage individuals to organise in a way that makes good solutions easier to achieve?

It is in this sense that space should be considered an enabling constraint. By limiting the degrees of freedom within a system in such a way as to encourage some types of reflexive and reciprocal interaction amongst its parts, space may steer system design into regimes that spontaneously exhibit useful order. This type of self-organisation, or "order for free", has been described carefully in the context of non-spatial networks [14], but has also been studied in natural systems where spatial organisation is foregrounded (e.g., [13]), and has even influenced the ideas of those trying to understand the nature of sound architectural form [1]. The contents of the current special issue are intended as a contribution to further elucidating these issues.

\section{Contents of this Special issue}

To begin, a review paper by Evans provides an overview of how the structure, dynamics and evolution of spatially embedded networks have been approached using the tools of network theory, and identifies some of the open challenges that face researchers seeking to understand real-world complex spatial networks. 
The following two papers investigate general models of spatially embedded random networks in which connectivity is influenced by spatial separation [3]. Bullock et al. explore the effect of structural constraints arising from spatial embedding on several network properties including giant component formation, degree correlation and the presence of scale free and small world properties. Buckley et al. focus on the effect of spatial embedding on the dynamic complexity of networks. They investigate a measure of complexity inspired by the balance between modularity and integration in neural systems $[20,2]$ and demonstrate how the constraints arising from spatial embedding increase interaction complexity relative to comparable non-spatial networks.

The next two papers consider the effects of spatial embedding on artificial and biological neural networks. Husbands et al. explore the influence of spatial and temporal constraints on a class of artificial neural networks known as GasNets, that incorporate two interacting signalling mechanisms: one based on electrical connections, the other on a diffusible gaseous neurotransmitter. Their findings indicate that spatial embedding facilitates exploitation of loose coupling between these signalling mechanisms in a way that enhances evolvability. Womble and Cohen investigate a spiking neural network model in which the distribution of time delays between neurons are governed by their spatial separation. They find that the introduction of spatial embedding reverses the effect of time delays, producing more oscillatory behaviour as time delays decrease, rather than more stationary behaviour, as is the case in non-spatial networks.

Finally, Batty considers the observation that size distributions of many human systems, such as cities and firms, tend to exhibit self-similarity in space and time, and can be described by powers laws that exhibit little change in scaling even over long periods of time. Using 'rank clock' visualisations, he reveals the micro-level volatility that underlies these apparently stable macro-level size distributions.

\section{Conclusions}

The ubiquity of spatial embedding in complex systems appears, paradoxically, to have reduced the extent to which complexity science has sought to understand its impact. While there are many studies that have modelled complex systems as spatially extended, they rarely attempt to isolate and understand the contribution that spatial embedding makes to the structural and functional organisation of the complex systems that we are striving to 
understand. We hope that the contents of this special issue are a step in this interesting direction.

\section{Acknowledgements}

Bullock and Geard acknowledge the support of research funding from the UK's Engineering and Physical Sciences Research Council (EP/C513711/1 and EP/D00232X/1, respectively).

\section{References}

[1] Alexander, C., 2004. The Nature of Order. CES Publishing.

[2] Barnett, L., Buckley, C. L., Bullock, S., 2009. Neural complexity and structural connectivity. Phys. Rev. E 79 (5), 051914.

[3] Barnett, L., Di Paolo, E., Bullock, S., 2007. Spatially embedded random networks. Phys. Rev. E 76 (5), 056115.

[4] Batty, M., 2005. Cities and Complexity. MIT Press.

[5] Boerlijst, M. C., Hogeweg, P., 1991. Spiral wave structures in pre-biotic evolution: Hypercycles stable against parasites. Physica D 48, 17-28.

[6] Buckley, C. L., Bullock, S., this volume. Spatially embedded dynamics and complexity. Complexity.

[7] Bullock, S., Buckley, C. L., 2009. Embracing the "tyranny of distance": Space as an enabling constraint. Technoetic Arts 7, 141-152, special issue on Living Buildings: Plectic Systems Architecture.

[8] Clark, B., Bullock, S., 2007. Shedding light on plant competition: Modelling the influence of plant morphology on light capture (and vice versa). Journal of Theoretical Biology (2), 208-217.

[9] Di Paolo, E. A., 2000. Ecological symmetry breaking can favour the evolution of altruism in an action-response game. Journal of Theoretical Biology 203, 135-152.

[10] Dueñas-Osorio, L., Craig, J. I., Goodno, B. J., Bostrom, A., 2007. Interdependent response of networked systems. Journal of Infrastructure Systems 13 (3), 185-194. 
[11] Eigen, M., Schuster, P., 1979. The Hypercycle: A Principle of Natural Self-organization. Springer.

[12] Fleckenstein, K. S., 2005. Faceless students, virtual places: Emergence and communal accountability in online classrooms. Computers and Composition 22 (2), 149 - 176.

[13] Goodwin, B., 1994/2001. How the Leopard Changed its Spots. Princeton University Press.

[14] Kauffman, S., 1993. The Origins of Order. Oxford University Press, Oxford.

[15] Ladley, D., Bullock, S., 2004. Logistic constraints on 3D termite construction. In: Dorigo, M., Birattari, M., Blum, C., Gambardella, L. M., Mondada, F., Stützle, T. (Eds.), Fourth International Workshop on Ant Colony Optimization and Swarm Intelligence. Springer-Verlag, Berlin, pp. 178-189.

[16] Ladley, D., Bullock, S., 2005. The role of logistic constraints in termite construction of chambers and tunnels. Journal of Theoretical Biology $234,551-564$.

[17] Miller, W. L., Crabtree, B. F., McDaniel, R., Stange, K. C., 1998. Understanding change in primary care practice using complexity theory. Journal of Family Practice 46 (5), 369-376.

[18] Quayle, A., Bullock, S., 2006. Modelling the evolution of genetic regulatory networks. Journal of Theoretical Biology 238 (4), 737.

[19] Sauer, P. S. K., Davies, D. G., Costerton, J. W., 2002. Biofilms as complex differentiated communities. Annual Review of Microbiology 56, 187-209.

[20] Tononi, G., Sporns, O., Edelman, G. M., 1994. A measure for brain complexity: Relating functional segregation and integration in the nervous system. Proc. Natl. Acad. Sci. 91, 5033-5037. 